\title{
Long-term follow-up of intensive chemotherapy followed by reduced-dose and reduced-field irradiation for intracranial germ cell tumor
}

\author{
Akinori Takada, MD, ${ }^{1}$ Noriko li, MD, PhD, ${ }^{2}$ Masahiro Hirayama, MD, PhD, ${ }^{3}$ Hidemi Toyoda, MD, PhD, ${ }^{3}$ \\ Toshio Matsubara, MD, PhD, ${ }^{4}$ Yutaka Toyomasu, MD, ${ }^{1}$ Tomoko Kawamura, MD, ${ }^{1}$ \\ Takashi Daimon, PhD, ${ }^{5}$ Hajime Sakuma, MD, PhD, ${ }^{6}$ and Yoshihito Nomoto, MD, PhD ${ }^{7}$
}

Departments of ${ }^{1}$ Radiation Oncology, ${ }^{3}$ Pediatrics, and ${ }^{4}$ Neurosurgery, Mie University Hospital, Tsu City, Mie; ${ }^{2}$ Department of Radiation Oncology, Ise Red Cross Hospital, Ise City, Mie; ${ }^{5}$ Department of Biostatistics, Hyogo College of Medicine, Nishinomiya City, Hyogo; and Departments of ${ }^{6}$ Radiology and ${ }^{7}$ Radiation Oncology, Mie University Graduate School of Medicine, Tsu City, Mie, Japan

OBJECTIVE The authors analyzed the efficacy of intensive chemotherapy followed by reduced-dose and reduced-field irradiation for intracranial germ cell tumors (GCTs) and evaluated the long-term late effects caused by chemoradiotherapy (CRT).

METHODS The authors performed a retrospective study. The subjects were 24 patients who received CRT between April 1994 and April 2015. After surgery, intensive chemotherapy followed by reduced-dose and reduced-field irradiation was administered. For those with pure germinoma, who comprised the "good prognosis" group, five courses of conventional-dose chemotherapy (CDC) were administered, and radiotherapy (24 Gy) was applied to the whole ventricle. For all others, defined as the "intermediate and poor prognosis" group, two or three courses of CDC and high-dose chemotherapy were administered with peripheral blood stem cell transplantation and radiotherapy (24-30 Gy) applied to the whole ventricle or a larger field with or without local boost irradiation (20 Gy), which was applied as needed.

RESULTS The median period of follow-up was 112.5 months (range 28-261 months), and the 5-/10-year overall and progression-free survival rates were $100 \% / 83.5 \%$ and $91.3 \% / 86.5 \%$, respectively. The 5 -/10-year overall survival rates determined based on the histological subtypes were $100 \% / 100 \%$ for pure germinoma and $93.8 \% / 78.7 \%$ for others, respectively. The late toxicities were as follows: endocrine disorder ( $33 \%$ in pure germinoma, $56 \%$ in others), involuntary movements (17\% in pure germinoma, $39 \%$ in others), ear and labyrinth disorders (17\% in pure germinoma, $33 \%$ in others), and psychiatric disorders ( $0 \%$ in pure germinoma, $33 \%$ in others). Nineteen of 24 patients underwent MRI (T2*- or susceptibility-weighted imaging) after radiotherapy, and 16 (84\%) of those 19 patients had microbleeds detected, while 2 $(10.5 \%)$ had radiation-induced cavernous vascular malformations detected.

CONCLUSIONS Intensive chemotherapy followed by reduced-dose and reduced-field irradiation for intracranial GCTs had the same outcome as that reported in the literature, but late adverse effects after treatment were observed. Almost all of the complications were relatively mild but had the potential to lead to psychiatric disorders and intracranial hemorrhaging.

https://thejns.org/doi/abs/10.3171/2018.9.PEDS18181

KEYWORDS intracranial germ cell tumor; chemoradiotherapy; late toxicity; oncology

I NTRACRANIAL germ cell tumors (GCTs) represent approximately $3 \%$ of all pediatric CNS tumors and are more frequent in Japan than in Western countries. ${ }^{8,26}$ Intracranial GCTs are classified into two categories according to the histological subtype: germinomas and non- germinomatous GCTs (NGGCTs) in the WHO classification system. ${ }^{28}$ NGGCTs include embryonal carcinoma, yolk sac tumor, choriocarcinoma, teratoma, and mixed tumors. Another classification system separates intracranial GCTs into 3 risk groups: "good prognosis" (pure ger-

ABBREVIATIONS AFP = alpha-fetoprotein; CDC = conventional-dose chemotherapy; $C M B=$ cerebral microbleed; $C R T=$ chemoradiotherapy; $C S I=$ craniospinal irradiation; EP = etoposide and cisplatin; GCT = germ cell tumor; HCG = human chorionic gonadotropin; HDC = high-dose chemotherapy; ICE = ifosfamide, cisplatin, and etoposide; NGGCT = nongerminomatous GCT; OS = overall survival; PBSCT = peripheral blood stem cell transplantation; PFS = progression-free survival; RICM = radiationinduced cavernous malformation; STGC = syncytiotrophoblastic giant cell; SWI = susceptibility-weighted imaging.

SUBMITTED March 26, 2018. ACCEPTED September 6, 2018.

INCLUDE WHEN CITING Published online November 23, 2018; DOI: 10.3171/2018.9.PEDS18181. 
TABLE 1. Characteristics of the 24 patients included in the study

\begin{tabular}{lc}
\hline \multicolumn{1}{c}{ Variable } & Value \\
\hline Treatment period & $4 / 1994$ to $4 / 2015$ \\
\hline Median observational period (range), mos & $112.5(28-261)$ \\
\hline Sex, $\mathrm{n}(\%)$ & $20(83)$ \\
\hline Males & $4(17)$ \\
\hline Females & $13(1-34)$ \\
\hline Median age (range), yrs & \\
\hline Location, $\mathrm{n}(\%)$ & $10(42)$ \\
\hline Pineal body & $3(13)$ \\
\hline Suprasellar & $6(25)$ \\
\hline Nucleus basalis, brainstem & $5(21)$ \\
\hline Multifocal & $4(17)$ \\
\hline With dissemination & $8(33)$ \\
\hline Surgery, $\mathrm{n}(\%)$ & $6(25)$ \\
\hline None & $8(33)$ \\
\hline Biopsy & $2(8)$ \\
\hline Partial excision & \\
\hline Total excision & $6(25)$ \\
\hline Classification of histological subgroups, $\mathrm{n}(\%)$ & $18(75)$ \\
\hline Pure germinoma & $12(50)$ \\
\hline Others & $5(21)$ \\
\hline Germinoma w/ STGC & $1(4)$ \\
\hline Mixed GCT & \\
\hline Choriocarcinoma & \\
\hline
\end{tabular}

minoma and mature teratoma), "intermediate prognosis" (germinoma with syncytiotrophoblastic giant cell [STGC], immature teratoma, teratoma with malignant transformation, and mixed tumors composed mainly of germinoma or teratoma), and "poor prognosis" (choriocarcinoma, yolk sac tumor, embryonal carcinoma, and mixed tumors composed mainly of choriocarcinoma, yolk sac tumor, or embryonal carcinoma). ${ }^{20}$ These classifications reflect the overall survival (OS) of patients. The 5-year OS rate for pure germinoma is more than $90 \%$, while the 5-year OS rate for NGGCTs is lower, ranging from $33.6 \%$ to $68 \% .^{12,21}$ The majority of patients have a relatively long life expectancy.

Intracranial GCTs are sensitive to both radiotherapy and chemotherapy, regardless of their histology; however, treatment with chemotherapy alone has resulted in unacceptable tumor recurrence rates.${ }^{16}$ Regarding radiotherapy, intracranial GCTs have a tendency to spread via CSF, and therefore craniospinal irradiation (CSI) plus a boost has historically been considered the gold-standard treatment. However, this approach involves a risk of serious late toxicities such as learning disorders, endocrine defects, and growth disturbance, as the majority of patients with intracranial GCTs are children. ${ }^{13,14,17}$ In addition, cerebrovascular accidents are also a critical issue for long-surviving patients who received intracranial irradiation. ${ }^{4}$ Therefore, several clinical studies have described the application of intensive chemotherapy followed by reduced-dose and reduced-field irradiation to reduce the incidence of late tox- icity. ${ }^{22}$ Late toxicities have been reported to occur around a median follow-up of 2 years. Most reports about the long-term therapeutic toxicities of intracranial GCTs have included the toxicities caused by not only chemoradiotherapy (CRT) but also radiotherapy alone., ${ }^{1,18}$ Few studies have performed the long-term evaluation of late effects with respect to the recent modified CRT regimen.

Owing to recent developments in MRI techniques, radiation-induced small vessel disease such as cerebral microbleeds (CMBs) and radiation-induced cavernous malformations (RICMs) are now being detected..$^{24}$ Roddy et al. reported that in survivors of pediatric brain tumors who received cranial radiation therapy, CMBs were common and associated with neurocognitive deficits. ${ }^{27} \mathrm{It}$ is therefore important to understand the late effects that might affect patients treated with the recent modified CRT regimen.

In the present study, we retrospectively analyzed late effects of intensive chemotherapy followed by reduceddose and reduced-field irradiation for intracranial GCTs. In particular, we focused on the late effects and the presence of CMBs in patients with pure germinoma (classified as the "good prognosis" group) and other patients (classified as the "intermediate and poor prognosis" group) from the data of long-term follow-up at Mie University Hospital.

\section{Methods}

We performed a retrospective study of patients with intracranial GCTs. The study population was 24 consecutive patients (20 males and 4 females) who were treated by CRT between April 1994 and April 2015 in our institution. The characteristics of the patients are summarized in Table 1 . The subjects were patients who could be followed for more than 2 years after treatment. The median age was 13 years (range 1-34 years), and the primary sites included the pineal body $(n=10,42 \%)$, the suprasellar region $(n=3$, $13 \%$ ), other regions (nucleus basalis, brainstem, and brain ventricle; $n=6,25 \%)$, and multifocal regions $(n=5,21 \%)$. Disseminated tumors were present in 4 patients (17\%), and 12 patients $(50 \%)$ were diagnosed with hydrocephalus before treatment. The serum and CSF levels of human chorionic gonadotropin (HCG) $-\beta$ and alpha-fetoprotein (AFP), and the cytology of the CSF were examined. Among the 24 patients, biopsy was performed in 6 cases $(25 \%)$ and resection was performed in 10 cases (41\%). The histopathology at the diagnosis was available for 16 patients $(67 \%)$, while $8(33 \%)$ of 24 patients were diagnosed clinically based on clinical examinations and neuroimaging.

\section{Treatment}

When pure germinoma was suspected based on examinations of tumor markers and imaging findings, a biopsy was performed. For NGGCTs, resection was conducted. In some cases when a tumor was located on the neurohypophysis in the suprasellar region and the tumor blood flow was abundant according to images, we avoided a biopsy or excision.

After surgery for a biopsy or excision, intensive chemotherapy followed by reduced-dose and reduced-field irradiation was administered. For pure germinoma (good prognosis group), 5 courses of conventional-dose chemo- 
therapy (CDC) were administered, and radiotherapy (24 Gy) was applied to the whole ventricle. For all others (intermediate and poor prognosis group), 2 or 3 courses of CDC and high-dose chemotherapy (HDC) were administered with peripheral blood stem cell transplantation (PBSCT), and radiotherapy (24-30 Gy) was applied to the whole ventricle or a larger field with or without local boost irradiation (20 Gy), which was applied as needed. In general, the radiation dose decreased during the study period.

Radiotherapy was delivered 5 days a week using a 6- to 10-MV linear accelerator. Daily fraction sizes of 1.8-2.0 and 1.5-1.8 Gy were used for the primary tumor and cerebrospinal axis, respectively. In the early cases, the treatment fields were determined using conventional $\mathrm{x}$-ray simulators and, more recently, 3D conformal radiotherapy. The radiation field and dose varied according to the histopathology, tumor extent, patient age, CSF results, spinal MRI findings, and serum HCG- $\beta$ or AFP values.

\section{Statistical Analyses}

We used the Kaplan-Meier method to calculate the OS and progression-free survival (PFS) rates for the analysis of the treatment outcomes. The OS was calculated from the date on which radiotherapy started until the date of the last follow-up examination or death. The PFS was calculated from the date on which radiotherapy started until the date of the last follow-up examination or death or relapse. Statistical analyses were performed using $\mathrm{R}$ statistical software (version 3.2.3, R core team, 2014; R Foundation for Statistical Computing).

\section{Ethical Considerations}

All study participants provided informed consent, and the study design was approved by the appropriate ethics review boards.

\section{Results}

\section{Patient Characteristics}

Six $(25 \%)$ of 24 patients with pure germinoma were classified into the good prognosis group, and 18 (75\%) with other lesions were classified into the intermediate and poor prognosis group (germinoma with STGC, $\mathrm{n}=12$; mixed GCT, $\mathrm{n}=5$; and choriocarcinoma, $\mathrm{n}=1$; Table 1). The first symptoms of the patients were as follows: eye movement disorders (double vision, upward gaze disturbance; $\mathrm{n}=13$, $54 \%$ ); intracranial hypertension (headache, nausea, and vomiting; $\mathrm{n}=11,46 \%)$; diabetes insipidus $(\mathrm{n}=11,46 \%)$, motor impairment $(\mathrm{n}=3,13 \%)$, weight loss $(\mathrm{n}=1,4 \%)$, and convulsion $(\mathrm{n}=2,8 \%)$. Twelve $(50 \%)$ of the patients were diagnosed with hydrocephalus at the initial visit (Table 2).

The chemotherapy results are shown in Table 3. All 6 (100\%) of the patients with pure germinoma received CDC alone. HDC combined with CDC was applied to $11(61 \%)$ of the 18 patients with other lesions, and the rest received CDC alone.

The dosage and radiation fields for each histological subtype are specified in Table 4. All of the patients with pure germinoma $(n=6)$ received whole-ventricle field irradiation (24-30 Gy; median 24 Gy). In the other patients, the total dose was 24-50.4 Gy (median 45 Gy). Regarding
TABLE 2. Primary symptom and primary lesion

\begin{tabular}{lc}
\hline \multicolumn{1}{c}{ Primary symptom } & Value (\%) \\
\hline Oculomotor disturbance (double vision, upper gaze palsy) & $13(54)$ \\
\hline Intracranial hypertension (headache, nausea, vomiting) & $11(46)$ \\
\hline Diabetes insipidus & $11(46)$ \\
\hline Motor disorder & $3(13)$ \\
\hline Convulsion, impaired consciousness & $2(8)$ \\
\hline Dizziness, fatigue & $2(8)$ \\
\hline Irregular menstruation, amenorrhea & $2(8)$ \\
\hline Weight loss & $1(4)$ \\
\hline Hydrocephalus due to primary lesion, $n(\%)$ & $7 / 10(70)$ \\
\hline Pineal body & $1 / 3(33)$ \\
\hline Suprasellar (bulky) & $2 / 6(33)$ \\
\hline Others (basal ganglia, midbrain, cerebellum) & $2 / 5(40)$ \\
\hline Multifocal & $12(50)$ \\
\hline Hydrocephalus at the first visit & $6(25)$ \\
\hline Expanded a shunt
\end{tabular}

the radiation field, 3 patients received local field irradiation, 9 received whole-ventricle field irradiation, 3 received whole-brain field irradiation, and 3 received CSI. Secondlook surgery was performed in 1 non-complete response case after CRT.

\section{Survival Rate}

The median follow-up period was 112.5 months (range 28-261 months), and the 5-/10-year OS and PFS rates were $100 \% / 83.5 \%$ and $91.3 \% / 86.5 \%$, respectively (Fig. 1). The 5-/10-year OS rates determined based on the histological subtypes were $100 \% / 100 \%$ for pure germinoma and $93.8 \% / 78.7 \%$ for others, respectively, and the 5-/10year OS rates determined based on the primary site were $100 \% / 100 \%$ for suprasellar, $100 \% / 88.9 \%$ for pineal body, $100 \% / 80 \%$ for nucleus and brainstem, and $100 \% / 66.7 \%$ for multifocal lesion (Fig. 2).

Among all of the patients in this study, 3 with germinoma with STGC relapsed and died of their disease. One patient with pure germinoma had late recurrence at 132 months, more than 10 years after the completion of treatment.

\section{Late Toxicities}

Late toxicities were classified according to the Common Terminology Criteria for Adverse Events (version 4.0). In this study, 20 (83\%) of 24 patients experienced a certain degree of various late toxicities. It is difficult to distinguish between the symptoms caused by the tumor and those caused by treatment. The patients' primary symptoms were diabetes insipidus, eye movement disorders, or motor impairment; these symptoms persisted in almost all cases, even after treatment. Only 4 of 13 patients showed improvement in their eye movement disorders after treatment. We therefore did not include these symptoms among adverse events. In addition, 1 patient (17\%) in the pure germinoma group had an eating disorder as a symptom before 
TABLE 3. Chemotherapy regimens and tumor maker test

\begin{tabular}{|c|c|c|c|c|c|c|c|}
\hline $\begin{array}{l}\text { Case } \\
\text { No. }\end{array}$ & $\begin{array}{l}\text { Histological } \\
\text { Subtype }\end{array}$ & Surgery & Pathology & $\begin{array}{c}\text { HCG }-\beta \\
\text { (serum or CSF) }\end{array}$ & $\begin{array}{c}\text { AFP } \\
\text { (serum or CSF) }\end{array}$ & $\begin{array}{c}\text { CDC } \\
\text { (no. of courses) }\end{array}$ & $\mathrm{HDC}$ \\
\hline 1 & Others & Biopsy & Germinoma w/ STGC & Positive & Negative & PEB (4) & $1+$ auto BMT \\
\hline 2 & Others & Not done & Not done & Positive & Negative & PEB (3), JEB (2) & $3+$ auto PBSCT \\
\hline 3 & Others & Total excision & Germinoma w/ STGC & Positive & Positive & $\mathrm{EP}(4)$ & None \\
\hline 4 & Others & Partial excision & Germinoma w/ STGC & Positive & Negative & $\begin{array}{l}\text { CARE (2), EP } \\
\text { (1) }\end{array}$ & $2+$ auto PBSCT \\
\hline 5 & Others & Biopsy & Germinoma w/ STGC & Positive & Negative & ICE (3) & $3+$ auto PBSCT \\
\hline 6 & Others & Total excision & Mixed GCTs (immature teratoma) & Negative & Positive & ICE (1) & $2+$ auto PBSCT \\
\hline 7 & Others & Partial excision & $\begin{array}{l}\text { Mixed GCTs (germinoma w/ STGC } \\
+ \text { yolk sac tumor component) }\end{array}$ & Positive & Positive & CARE (2) & $1+$ auto PBSCT \\
\hline 8 & Pure germinoma & Partial excision & Pure germinoma & Negative & Negative & ICE (4) & None \\
\hline 9 & Others & Not done & Not done & Positive & Negative & $\operatorname{ICE}(3), \operatorname{EP}(5)$ & None \\
\hline 10 & Others & Not done & Not done & Positive & Negative & $\operatorname{ICE}(3)$ & $2+$ auto PBSCT \\
\hline 11 & Pure germinoma & Not done & Not done & Negative & Negative & ICE (5) & None \\
\hline 12 & Others & Biopsy & Germinoma w/ STGC & Positive & Negative & ICE (2) & $1+$ auto PBSCT \\
\hline 13 & Pure germinoma & Not done & Not done & Negative & Negative & ICE (5) & None \\
\hline 14 & Others & Partial excision & Choriocarcinoma & Positive & Negative & ICE (2) & $2+$ auto PBSCT \\
\hline 15 & Others & Partial excision & $\begin{array}{l}\text { Mixed GCTs (mature teratoma + } \\
\text { yolk sac tumor) }\end{array}$ & Negative & Positive & ICE (5) & None \\
\hline 16 & Others & Partial excision & Germinoma w/ STGC & Positive & Negative & ICE (2) & $3+$ auto PBSCT \\
\hline 17 & Pure germinoma & Biopsy & Pure germinoma & Negative & Negative & $\operatorname{ICE}(5)$ & None \\
\hline 18 & Pure germinoma & Partial excision & Pure germinoma & Negative & Negative & ICE (5) & None \\
\hline 19 & Others & Not done & Not done & Positive & Negative & ICE (2) & $2+$ auto PBSCT \\
\hline 20 & Others & Partial excision & Germinoma w/ STGC & Positive & Negative & $\operatorname{ICE}(5)$ & None \\
\hline 21 & Pure germinoma & Biopsy & Pure germinoma & Negative & Negative & ICE (3) & None \\
\hline 22 & Others & Biopsy & Germinoma w/ STGC & Positive & Negative & $\operatorname{ICE}(5)$ & None \\
\hline 23 & Others & Not done & Not done & Positive & Negative & ICE (5) & None \\
\hline 24 & Others & Second look & Mixed GCTs (mature teratoma) & Negative & Negative & ICE (4) & None \\
\hline
\end{tabular}

auto = autologous; BMT = bone marrow transplantation; CARE = carboplatin and etoposide; JEB = carboplatin, etoposide, and bleomycin; $\mathrm{PEB}=$ cisplatin, etoposide, and bleomycin.

treatment, and it did not improve after treatment. We also did not define this symptom as a late toxicity.

Table 5 shows the incidence of late toxicities in the pure germinoma and other patient groups in this study. The late toxicities were as follows: endocrine disorder, including hypothyroidism and delayed puberty (33\% in pure germinoma, $56 \%$ in others); involuntary movements, including extrapyramidal disorder and ataxia (17\% in pure germinoma, 39\% in others); ear and labyrinth disorders, including hearing impaired, tinnitus, and vertigo (17\% in pure germinoma, 33\% in others); seizure, including electroen-

\section{TABLE 4. Dosage and radiation fields}

\begin{tabular}{lcl}
\hline \multicolumn{1}{c}{$\begin{array}{c}\text { Histological } \\
\text { Subtype }\end{array}$} & $\begin{array}{c}\text { Median Dose } \\
\text { (range) }\end{array}$ & \multicolumn{1}{c}{ Field (no. of patients) } \\
\hline Pure germinoma & $24(24-30)$ & WV (6) \\
\hline Others & $45(24-50.4)$ & $\begin{array}{r}\text { Local (3); WV (1); WV initially, then } \\
\text { local (8); WB (3); CSI (1); CSI ini- } \\
\text { tially, then spinal cord + local (2) }\end{array}$ \\
\hline
\end{tabular}

$\mathrm{WB}=$ whole brain; $\mathrm{WV}=$ whole ventricle. cephalogram abnormality (17\% in pure germinoma, $33 \%$ in others); memory impairment and cognitive disturbance (17\% in pure germinoma, $28 \%$ in others); azoospermia ( $0 \%$ in pure germinoma, $6 \%$ in others); and psychiatric disorders, including school refusal, anxiety disorder, eating disorder, and self-injury (0\% in pure germinoma, 33\% in others). One patient in the "others" group was unable to reintegrate into society. None of the patients suffered from treatment-induced secondary neoplasm.

Three patients married after treatment and later had children. The dosages of the hypothalamic-pituitary unit and the characteristics of those 3 patients were as follows: 1 patient with pure germinoma received 24 Gy and had no hormonal disorder; 1 patient with other lesions received 50 Gy and had 2 children after treatment for her infertility; and 1 patient with other lesions received $42 \mathrm{~Gy}$, and despite being diagnosed with azoospermia, he recovered spontaneously and fathered a child.

All patients underwent MRI. Nineteen patients received $\mathrm{T} 2 *$-weighted gradient-echo imaging (T2*) or susceptibility-weighted imaging (SWI) during the follow-up. CMBs were detected in $16(84 \%)$ of the 19 patients (4 in the pure germinoma group and 12 in the others group), and RICMs 

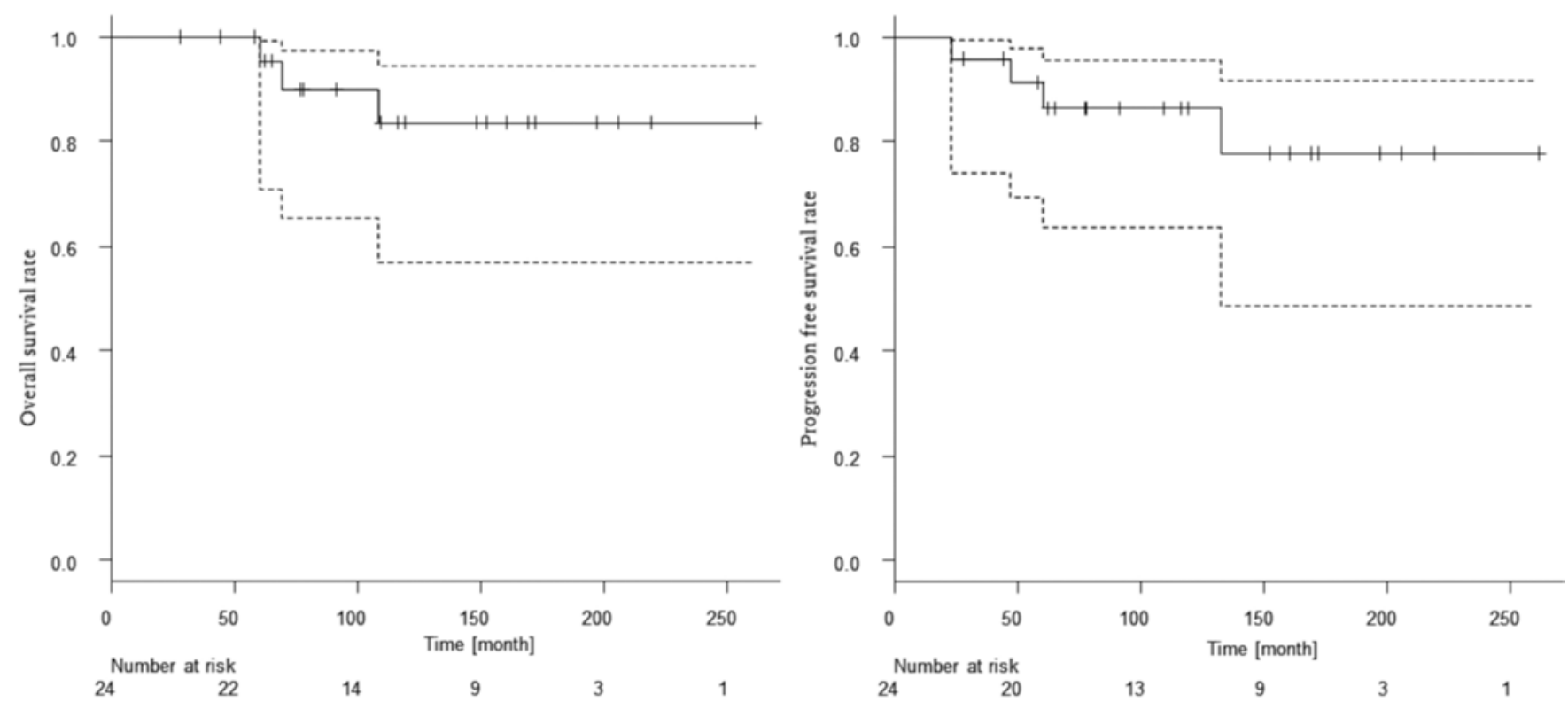

FIG. 1. Kaplan-Meier survival curves of the OS (left) and PFS (right) rates for all 24 patients. Dashed lines represent $95 \%$ confidence intervals.

were detected in 2 patients $(10.5 \%), 1$ in the pure germinoma group and 1 in the others group (Table 6). None had symptoms caused by RICMs.

\section{Discussion}

In the 1990s, CSI was the gold-standard therapy for intracranial GCTs, but the treatment was associated with severe late toxicities. Local field irradiation has led to unacceptably high rates of CNS recurrence, even in pa- tients who receive neoadjuvant chemotherapy. ${ }^{15}$ The most common approach to treating localized intracranial GCTs has now shifted toward treatment of the whole ventricular system followed by boost irradiation at the site of initial disease with or without chemotherapy. Aoyama et al. ${ }^{3}$ presented promising results of intracranial GCTs that were treated with surgery followed by chemotherapy with etoposide and cisplatin (EP), or ifosfamide, cisplatin, and etoposide (ICE) and low-dose involved-field radiotherapy. The 5-year OS and recurrence-free survival rates for pa-
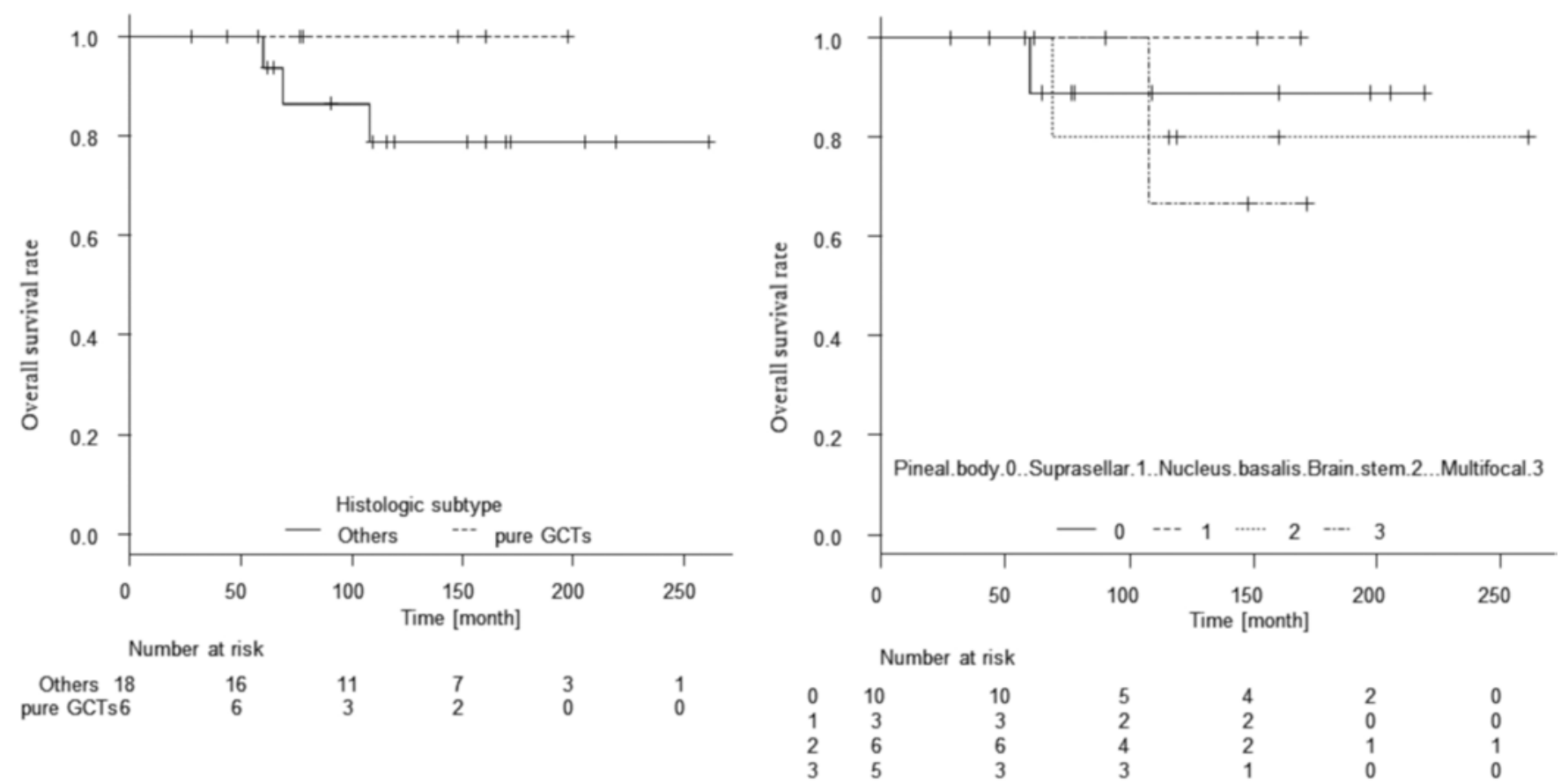

FIG. 2. Kaplan-Meier survival curves of the OS rates according to the histological subtype (left) and primary lesion (right). 
TABLE 5. Late toxicities of CRT grouped by histological subtype

\begin{tabular}{|c|c|c|c|c|c|c|c|c|}
\hline \multirow[b]{2}{*}{ Late Toxicity } & \multicolumn{4}{|c|}{ Pure Germinoma $(n=6)$} & \multicolumn{4}{|c|}{ Others $(n=18)$} \\
\hline & Grades 1-2 & Grade 3 & Grade 4 & Cases $(\%)$ & Grades 1-2 & Grade 3 & Grade 4 & Cases (\%) \\
\hline Endocrine disorder: hypothyroidism, delayed puberty & 2 & - & - & $2(33)$ & 10 & - & - & $10(56)$ \\
\hline Movements involuntary: extrapyramidal disorder, ataxia & 1 & - & - & $1(17)$ & 6 & 1 & - & $7(39)$ \\
\hline $\begin{array}{l}\text { Ear \& labyrinth disorders: hearing impaired, tinnitus, } \\
\text { vertigo }\end{array}$ & 1 & - & - & $1(17)$ & 5 & 1 & - & $6(33)$ \\
\hline Seizure: electroencephalogram abnormality & 1 & - & - & $1(17)$ & 6 & - & - & $6(33)$ \\
\hline Memory impairment, cognitive disturbance & 1 & - & - & $1(17)$ & 5 & - & - & $5(28)$ \\
\hline Azoospermia & 0 & - & - & $0(0)$ & - & 1 & - & $1(6)$ \\
\hline $\begin{array}{l}\text { Psychiatric disorders: school refusal, anxiety disorder, } \\
\text { eating disorder, self-injury, etc. }\end{array}$ & 0 & - & - & $0(0)$ & 5 & 1 & - & $6(33)$ \\
\hline
\end{tabular}

tients with pure germinoma were $100 \%$ and $86 \%$, while those for patients with germinoma with STGC were $75 \%$ and $44 \%$, respectively. ${ }^{3}$ Many studies have reported an excellent prognosis after EP and ICE regimens followed by radiotherapy. ${ }^{11,31}$ In the present study, we adopted ICE and whole-ventricle radiotherapy ( $\leq 30 \mathrm{~Gy}$ ) for the treatment of pure germinoma.

HDC with stem cell rescue has been reported to show promise for the treatment of relapsed GCTs. This approach has been considered for patients who do not experience a complete response following all other treatment modalities. ${ }^{9}$ We adopted CRT with HDC for patients with other lesions, who belonged to the intermediate and poor prognosis group. One patient with a poor prognosis underwent CDC and local irradiation, as he was a 1-year-old boy with Down syndrome.

The treatment protocol has been changed over time. We recently adopted CRT without HDC for patients with germinoma with STGC. The 10-year OS and PFS rates for patients with other lesions were $93 \%$ and $66 \%$, respectively. These data were almost the same as in other studies.

The role of surgery for germinoma has been discussed but remains controversial. Sawamura et al. reported that there were no significant differences in the outcome based on the extent of resection. ${ }^{29}$ However, Souweidane et al. ${ }^{32}$ reported that the successful treatment of children with primary CNS GCTs can be greatly influenced by the neurosurgeon involved in the diagnostic and therapeutic care of such children. Advances in neurosurgical management (microsurgical dissection, endoscopic neurosurgery, and navigation guidance) have made it possible to reduce the rate of serious adverse events, and the role of surgery is becoming increasingly important. ${ }^{32}$ Details of surgeries conducted in our hospital are shown in Table 1. When the tumor was located on the neurohypophysis in the suprasellar region, we did not perform surgery in order to avoid involvement of the pituitary gland or induce a hypothalamic disorder (obesity, weight loss, and emotional disorder).

With an increasing curative rate, improving the quality of life by reducing the rate of long-term complications induced by treatment will become the next major objective. Packer et al. reported that a radiation dose $\geq 30$ Gy to any cortical segment of the brain was associated with a 2-fold increased risk for late seizure disorder in children with brain tumors. ${ }^{23}$ Sawamura et al. investigated late toxicities after radiotherapy or CRT for intracranial GCTs and found that 58 of 85 patients required hormonal replacement therapy, and 26 patients required some support in their daily life. ${ }^{30}$ Radiotherapy tends to correlate with physical or cognitive sequelae. In the present study, all patients were treated with intensive chemotherapy followed by reduceddose and reduced-field irradiation. However, psychiatric disorders developed in 6 patients, one of which was grade 3 severity; that patient had hydrocephalus during HDC and later suffered from pyramidal tract syndrome and psychiatric disorder (anxiety disorder, adjustment disorder, selfinjurious behavior, etc.). Unfortunately, this patient has not been able to reintegrate into society. All patients who had psychiatric disorders had lesions other than pure germinoma, and none of the patients with pure germinoma had psychiatric disorders. A higher dose and wider field of radiation and HDC may be risk factors for psychiatric disorders. Furthermore, primary symptoms such as hearing disorders, cognitive disturbance, and eye movement disorders might lead to psychiatric disorders, particularly school refusal and anxiety disorder., 5

According to the 2013 guideline of the American Society of Clinical Oncology, gonadal toxicity with radiotherapy is of concern. Whole-brain irradiation exceeding 40 Gy and irradiation exceeding 15 Gy to the abdominal area or pelvis can produce infertility at a high rate of $70 \%$ or higher for both sexes. ${ }^{19}$ In our study, 3 patients married after treatment and subsequently had children. One patient who received 24 Gy had no hormonal disorder; the other 2 received more than $40 \mathrm{~Gy}$, and 1 required treatment for infertility. Depending on the dose, radiotherapy may carry a risk of infertility.

With advances in imaging techniques, CMBs and cavernomas can now be detected using either T2* or SWI during long-term follow-up after intracranial irradiation. These are described as microcirculatory disorders caused by radiotherapy. In the present study, CMBs were found in 16 patients (84\%) and RICMs in $2(10.5 \%)$. The incidence of CMBs in children treated with intracranial irradiation reportedly ranges from $29 \%$ to $100 \%,,^{24,25,33}$ with the rate increasing over time. However, there have been no reports of CMBs after low-dose irradiation for pure germinoma. In our cases, as noted above, 4 of 5 patients in the pure germinoma group presented with CMBs. Roddy et al. reported that whole-brain irradiation, exposure to chemo- 
TABLE 6. Results of MRI examination

\begin{tabular}{lccc}
\hline \multicolumn{1}{c}{ Variable } & Pure Germinoma & Others & Total \\
\hline No. of cases & 6 & 18 & 24 \\
\hline $\begin{array}{l}\text { No. who underwent an MRI } \\
\quad \text { examination (T2* or SWI) }\end{array}$ & 5 & 14 & 19 \\
\hline CMBs (\%) & $4(80)$ & $12(85.7)$ & $16(84.2)$ \\
\hline RICMs (\%) & $1(20)$ & $1(7.1)$ & $2(10.5)$ \\
\hline
\end{tabular}

therapy, and older age at intracranial irradiation were associated with an increased rate of CMB development, while the dose of radiation therapy was not significantly associated. ${ }^{27}$ Our results certainly agree with those results. For the patients in the pure germinoma group, whole-ventricle irradiation (which resembles whole-brain irradiation) and exposure to chemotherapy may cause high rates of $\mathrm{CMB}$ development despite low-dose irradiation. This group received only 24-30 Gy, but long-term follow-up revealed CMBs.

The spontaneous incidence of cavernomas was reported to be $0.6 \% .^{2}$ The incidence of RICMs $(10.5 \%)$ in our study was extremely high compared to the spontaneous incidence of cavernous malformations. While our patients had no clinical symptoms, Cutsforth-Gregory et al. reported that, in the absence of symptoms at presentation, the risk of hemorrhaging due to RICMs was higher than that for nonradiation-induced cavernous malformations $(4.2 \%$ vs $0.35 \%$, respectively). ${ }^{7}$ Gross et al. found that a prior history of hemorrhaging, brainstem location, and associated developmental venous anomalies were significant risk factors for symptomatic hemorrhaging in children with cerebral cavernous malformations. ${ }^{10}$ Therefore, we recommend the long-term observation of these patients by MRI.

Regarding late toxicities in patients with pure germinoma, all of the late adverse events were classified as grade $\leq 2$, and no adverse events have been detected at all in 2 patients. Even among the patients with other lesions, most of the late adverse events were classified as less than grade 2 severity. On comparing the occurrence rate of adverse events, the rate in the group with other lesions was higher than that in the patients with pure germinoma. These findings suggest that the dose and field size might influence the occurrence of adverse events, and we believe that adequate long-term observation is necessary to clarify the associated toxicities. The limitations associated with this study include not only its retrospective nature and the small number of patients but also the wide variety of treatment methods employed.

\section{Conclusions}

Using a retrospective analysis, we confirmed that intensive chemotherapy followed by reduced-dose and reducedfield irradiation for intracranial GCTs resulted in the same outcome reported in the literature, but late adverse events after treatment were observed during the long-term followup (median 112.5 months). Almost all of the complications were relatively mild but had the potential to lead to psychiatric disorders that would require the intervention of a psychiatrist. Although the relationship with the symptoms is still unclear, vascular disorder was detected at a high frequency by MRI after radiotherapy. Careful long-term follow-up after treatment is important.

\section{References}

1. Acharya S, DeWees T, Shinohara ET, Perkins SM: Long-term outcomes and late effects for childhood and young adulthood intracranial germinomas. Neuro Oncol 17:741-746, 2015

2. Al-Holou WN, O'Lynnger TM, Pandey AS, Gemmete JJ, Thompson BG, Muraszko KM, et al: Natural history and imaging prevalence of cavernous malformations in children and young adults. J Neurosurg Pediatr 9:198-205, 2012

3. Aoyama H, Shirato H, Ikeda J, Fujieda K, Miyasaka K, Sawamura Y: Induction chemotherapy followed by low-dose involved-field radiotherapy for intracranial germ cell tumors. J Clin Oncol 20:857-865, 2002

4. Armstrong GT, Liu Q, Yasui Y, Huang S, Ness KK, Leisenring W, et al: Long-term outcomes among adult survivors of childhood central nervous system malignancies in the Childhood Cancer Survivor Study. J Natl Cancer Inst 101:946-958, 2009

5. Brinkman TM, Bass JK, Li Z, Ness KK, Gajjar A, Pappo AS, et al: Treatment-induced hearing loss and adult social outcomes in survivors of childhood CNS and non-CNS solid tumors: Results from the St. Jude Lifetime Cohort Study. Cancer 121:4053-4061, 2015

6. Brinkman TM, Li C, Vannatta K, Marchak JG, Lai JS, Prasad PK, et al: Behavioral, social, and emotional symptom comorbidities and profiles in adolescent survivors of childhood cancer: a report from the Childhood Cancer Survivor Study. J Clin Oncol 34:3417-3425, 2016

7. Cutsforth-Gregory JK, Lanzino G, Link MJ, Brown RD Jr, Flemming KD: Characterization of radiation-induced cavernous malformations and comparison with a nonradiation cavernous malformation cohort. J Neurosurg 122:1214-1222, 2015

8. Echevarría ME, Fangusaro J, Goldman S: Pediatric central nervous system germ cell tumors: a review. Oncologist 13:690-699, 2008

9. Goldman S, Bouffet E, Fisher PG, Allen JC, Robertson PL, Chuba PJ, et al: Phase II trial assessing the ability of neoadjuvant chemotherapy with or without second-look surgery to eliminate measurable disease for nongerminomatous germ cell tumors: A Children's Oncology Group Study. J Clin Oncol 33:2464-2471, 2015

10. Gross BA, Du R, Orbach DB, Scott RM, Smith ER: The natural history of cerebral cavernous malformations in children. J Neurosurg Pediatr 17:123-128, 2016

11. Matsutani M: Combined chemotherapy and radiation therapy for CNS germ cell tumors-the Japanese experience. J Neurooncol 54:311-316, 2001

12. Haas-Kogan DA, Missett BT, Wara WM, Donaldson SS, Lamborn KR, Prados MD, et al: Radiation therapy for intracranial germ cell tumors. Int J Radiat Oncol Biol Phys 56:511-518, 2003

13. Horowitz MB, Hall WA: Central nervous system germinomas. A review. Arch Neurol 48:652-657, 1991

14. Jennings MT, Gelman R, Hochberg F: Intracranial germcell tumors: natural history and pathogenesis. J Neurosurg 63: $155-167,1985$

15. Jensen AW, Laack NN, Buckner JC, Schomberg PJ, Wetmore CJ, Brown PD: Long-term follow-up of dose-adapted and reduced-field radiotherapy with or without chemotherapy for central nervous system germinoma. Int J Radiat Oncol Biol Phys 77:1449-1456, 2010

16. Kellie SJ, Boyce H, Dunkel IJ, Diez B, Rosenblum M, Brualdi L, et al: Primary chemotherapy for intracranial nongerminomatous germ cell tumors: results of the second inter- 
national CNS germ cell study group protocol. J Clin Oncol 22:846-853, 2004

17. Kiltie AE, Gattamaneni HR: Survival and quality of life of paediatric intracranial germ cell tumour patients treated at the Christie Hospital, 1972-1993. Med Pediatr Oncol 25:450-456, 1995

18. Liang SY, Yang TF, Chen YW, Liang ML, Chen HH, Chang KP, et al: Neuropsychological functions and quality of life in survived patients with intracranial germ cell tumors after treatment. Neuro Oncol 15:1543-1551, 2013

19. Loren AW, Mangu PB, Beck LN, Brennan L, Magdalinski AJ, Partridge AH, et al: Fertility preservation for patients with cancer: American Society of Clinical Oncology clinical practice guideline update. J Clin Oncol 31:2500-2510, 2013

20. Matsutani M, Sano K, Takakura K, Fujimaki T, Nakamura $\mathrm{O}$, Funata N, et al: Primary intracranial germ cell tumors: a clinical analysis of 153 histologically verified cases. J Neurosurg 86:446-455, 1997

21. Matsutani M, Takakura K, Sano K: Primary intracranial germ cell tumors: pathology and treatment. Prog Exp Tumor Res 30:307-312, 1987

22. O’Neil S, Ji L, Buranahirun C, Azoff J, Dhall G, Khatua S, et al: Neurocognitive outcomes in pediatric and adolescent patients with central nervous system germinoma treated with a strategy of chemotherapy followed by reduced-dose and volume irradiation. Pediatr Blood Cancer 57:669-673, 2011

23. Packer RJ, Gurney JG, Punyko JA, Donaldson SS, Inskip PD, Stovall M, et al: Long-term neurologic and neurosensory sequelae in adult survivors of a childhood brain tumor: childhood cancer survivor study. J Clin Oncol 21:3255-3261, 2003

24. Passos J, Nzwalo H, Marques J, Azevedo A, Netto E, Nunes $\mathrm{S}$, et al: Late cerebrovascular complications after radiotherapy for childhood primary central nervous system tumors. Pediatr Neurol 53:211-215, 2015

25. Peters S, Pahl R, Claviez A, Jansen O: Detection of irreversible changes in susceptibility-weighted images after wholebrain irradiation of children. Neuroradiology 55:853-859, 2013

26. Poynter JN, Fonstad R, Tolar J, Spector LG, Ross JA: Incidence of intracranial germ cell tumors by race in the United States, 1992-2010. J Neurooncol 120:381-388, 2014

27. Roddy E, Sear K, Felton E, Tamrazi B, Gauvain K, Torkildson J, et al: Presence of cerebral microbleeds is associated with worse executive function in pediatric brain tumor survivors. Neuro Oncol 18:1548-1558, 2016
28. Rosenblum MK, Nakazato Y, Matsutani M: CNS germ cell tumors, in: WHO Classification of Tumours of the Central Nervous System, ed 3. Albany, NY: WHO Publication Center, 2007, p 197

29. Sawamura Y, de Tribolet N, Ishii N, Abe H: Management of primary intracranial germinomas: diagnostic surgery or radical resection? J Neurosurg 87:262-266, 1997

30. Sawamura Y, Ikeda J, Shirato H, Tada M, Abe H: Germ cell tumours of the central nervous system: treatment consideration based on 111 cases and their long-term clinical outcomes. Eur J Cancer 34:104-110, 1998

31. Sawamura Y, Shirato H, Ikeda J, Tada M, Ishii N, Kato T, et al: Induction chemotherapy followed by reduced-volume radiation therapy for newly diagnosed central nervous system germinoma. J Neurosurg 88:66-72, 1998

32. Souweidane MM, Krieger MD, Weiner HL, Finlay JL: Surgical management of primary central nervous system germ cell tumors: proceedings from the Second International Symposium on Central Nervous System Germ Cell Tumors. J Neurosurg Pediatr 6:125-130, 2010

33. Yeom KW, Lober RM, Partap S, Telischak N, Tsolinas R, Barnes PD, et al: Increased focal hemosiderin deposition in pediatric medulloblastoma patients receiving radiotherapy at a later age. J Neurosurg Pediatr 12:444-451, 2013

\section{Disclosures}

The authors report no conflict of interest concerning the materials or methods used in this study or the findings specified in this paper.

\section{Author Contributions}

Conception and design: Takada. Acquisition of data: Takada, Ii, Hirayama, Toyoda, Matsubara, Toyomasu, Kawamura. Analysis and interpretation of data: Takada, Ii. Drafting the article: Takada, Ii, Hirayama, Toyoda, Matsubara, Toyomasu, Kawamura, Nomoto. Critically revising the article: Takada, Ii, Nomoto. Reviewed submitted version of manuscript: Takada. Approved the final version of the manuscript on behalf of all authors: Takada. Statistical analysis: Daimon. Study supervision: Hirayama, Sakuma, Nomoto.

\section{Correspondence}

Akinori Takada: Mie University Hospital, Tsu City, Mie, Japan. a-takada@clin.medic.mie-u.ac.jp. 